Methods To study the benefits of Darbepoetin and Pegfilgrastim in patients with de-compensated liver cirrhosis. Prospectively clinical data recorded since October 2014. Patients with active bleed, hepatorenal syndrome, hepatoma, portal vessel thrombosis were excluded. Patients started on Injection Darbepoetin alpha 200 microgram and Injection Pegfilgrastim $6 \mathrm{mg}$ subcutaneously every 15 days, total of three visits of patients, then three months follow up planned. Improvement in clinical, laboratory parameters analyzed. Median calculated, Wilcoxon Signed-Rank Test applied to compare both groups.

Results $\mathrm{N}=22$ all male, 3 lost to follow up, aetiology of cirrhosis were Non-alcoholic Fatty Liver Disease 5, Hepatitis B Virus 3, Hepatitis C Virus 2 and Alcohol 12. Median age 59years (range: 40 to 70 ). Improvement in Haemoglobin from 10.1 gram $\%$ (range $5.9-13.4 \mathrm{gm} \%$ ) to $10.6 \mathrm{gm} \%$ (range: $7.5-13.7$ gm\%) p value 0.00374 , Total leukocyte count from $5100 / \mathrm{cu}$ $\mathrm{mm}$ to $7100 / \mathrm{cu} \mathrm{mm} \mathrm{p}$ value 0.00214 , Platelet count $90,000 / \mathrm{cu}$ $\mathrm{mm}$ to $146,000 / \mathrm{cu} \mathrm{mm}, \mathrm{p}$ value 0.00096 , INR 1.7 (range 1.54.8 ) to $1.4 \mathrm{p}$ value 0.00064 , Albumin $2.4 \mathrm{gram} / \mathrm{dl}$ (range 1.62.9 ) to $2.5 \mathrm{gm} / \mathrm{dl}$ (range $1.8-3.5$ ) $\mathrm{P}$ value 0.043 , Child score from 10 to $8 \mathrm{P}$ value 0.007 , ascites score 2 to $1, \mathrm{P}$ value 0.00222 . No significant improvement in Serum creatinine, sodium, potassium, calcium, bilirubin, total protein and hepatic encephalopathy. High cost of medicine was the limiting factor. Conclusions Our study suggests that Darbepoetin Alpha and Pegfilgrastim is significantly effective in improving hematology, International normalized ratio, Albumin, ascites and Child score of liver cirrhosis patients.

\section{IDDF2019-ABS-0210 PRELIMINARY EFFICACY AND SAFETY OF 8-WEEK GLECAPREVIR/PIBRENTASVIR IN PATIENTS WITH HCV GENOTYPE 1-6 INFECTION AND COMPENSATED CIRRHOSIS: THE EXPEDITION-8 STUDY}

\begin{abstract}
${ }^{1}$ Robert S Brown Jr*, ${ }^{2}$ Christopher Hezode, ${ }^{3}$ Stanley Wang, ${ }^{4}$ Maria Buti, ${ }^{5}$ WanLong Chuang, ${ }^{6}$ Humberto Aguilar, ${ }^{7}$ Gabor Horvath, ${ }^{8}$ Barbara Rosado Carrion, ${ }^{9}$ Federico Rodriguez-Perez, ${ }^{3}$ Eric Cohen, ${ }^{3}$ Yiran B Hu, ${ }^{3}$ Gretja Schnell, ${ }^{3}$ Chih-Wei Lin, ${ }^{3}$ Lino Rodrigues, ${ }^{3}$ Roger Trinh, ${ }^{3}$ Federico J Mensa. ${ }^{1}$ Weill Cornell Medical College, Center for Liver Disease and Transplantation, USA; ${ }^{2}$ Department of Hepatology, Hôpital Henri Mondor, Université Paris-Est, Paris, France; ${ }^{3}$ AbbVie Inc., North Chicago, Illinois, USA; ${ }^{4}$ Vall d'Hebron University Hospital and CiBERHED del Instituto Carlos III, Barcelona, Spain; ${ }^{5}$ Kaohsiung Medical University Hospital, Kaohsiung Medical University, Kaohsiung, Taiwan; ${ }^{6}$ Louisiana Research Center, Shreveport, LA, USA; ${ }^{7}$ Hepatology Center of Buda, Budapest, Hungary; ${ }^{8}$ Director of GHGCPR Research Institute, Puerto Rico; ${ }^{9}$ GHWC and Gastroenterology Section, San Juan, Puerto Rico
\end{abstract}

10.1136/gutjnl-2019-IDDFabstracts.284

Background The pangenotypic direct-acting antivirals glecaprevir (identified by AbbVie and Enanta) coformulated with pibrentasvir $(\mathrm{G} / \mathrm{P})$ are approved to treat chronic HCV genotype (GT) 1-6 infection. Eight-week G/P achieved high SVR12 rates in Phase 2 and 3 studies, but was not studied in patients with compensated cirrhosis. Based on the high SVR12 rates demonstrated in treatment-naive patients with HCV GT1-6 infection and compensated cirrhosis treated with 12-week G/P, this study evaluates the efficacy and safety of an 8 -week $\mathrm{G} / \mathrm{P}$ treatment duration in that population. Methods EXPEDITION-8 is an ongoing phase 3, non-randomized, single arm, open-label, multicenter study conducted in adults with chronic HCV GT1-6 infection with compensated cirrhosis who are HCV treatment-naive. A recently approved protocol amendment enabled the inclusion of HCV GT3infected patients, who are not included in this analysis. G/P (300 mg/120 mg) is being dosed orally once-daily with food for 8 weeks. The primary efficacy endpoint is the SVR12 rate. Secondary endpoints are the on-treatment virologic failure and relapse rates. Adverse events and clinical laboratory abnormalities are being monitored in all patients.

Results In total, 280 treatment-naive patients with compensated cirrhosis have enrolled and are included in the analysis. To date, 116 patients have completed the post-treatment week 12 visit; (figure 1) shows preliminary efficacy results for those with available post-treatment week 4 and/or 12 data. No virologic failures have occurred. Adverse events (AEs) have been mostly mild, with the most common (at least 5\%) AEs being pruritus and fatigue (both 9\%), headache $(7 \%)$ and nausea (6\%). No AEs have led to discontinuation of G/P; 5 serious AEs have occurred, none of which were deemed related to $\mathrm{G} / \mathrm{P}$.

Conclusions In this ongoing study, G/P for 8 weeks in treatment-naive patients with $\mathrm{HCV}$ infection and compensated cirrhosis has been well-tolerated and achieved high rates of SVR, with no virologic failures to date. Updated efficacy and safety data will be presented at the meeting.

\section{IDDF2019-ABS-0211 EFFICACY AND SAFETY OF GLECAPREVIR/ PIBRENTASVIR IN PATIENTS WITH HCV GENOTYPE 5 OR 6 INFECTION: AN INTEGRATED ANALYSIS OF PHASE 2 AND 3 STUDIES}

${ }^{1}$ Betty B Yao*, ${ }^{2}$ Tarik Asselah, 'Linda Frederick, ${ }^{1}$ Gretja Schnell, ${ }^{3}$ Kris Kowdley, ${ }^{4}$ Paul Y Kwo, ${ }^{5}$ Fred Poordad, ${ }^{6}$ Kinh Nguyen, ${ }^{7}$ Samuel S Lee. ${ }^{1}$ AbbVie Inc., North Chicago, Illinois, USA; ${ }^{2}$ Centre de Recherche sur I'Inflammation, INSERM UMR 1149, Universite Paris Diderot, Department of Hepatology, AP-HP Hôpital Beaujon, Clichy, France; ${ }^{3}$ Swedish Medical Center, Seattle, USA; ${ }^{4}$ Stanford University School of Medicine, Palo Alto, CA, USA; ${ }^{5}$ The Texas Liver Institute, University of Texas Health, San Antonio, TX, USA; ${ }^{6}$ National Hospital for Tropical Diseases, Vietnam; ' University of Calgary, Calgary, AB, Canada

\subsection{6/gutjnl-2019-IDDFabstracts.285}

Background The pangenotypic direct-acting antivirals (DAAs) glecaprevir (identified by AbbVie and Enanta) coformulated

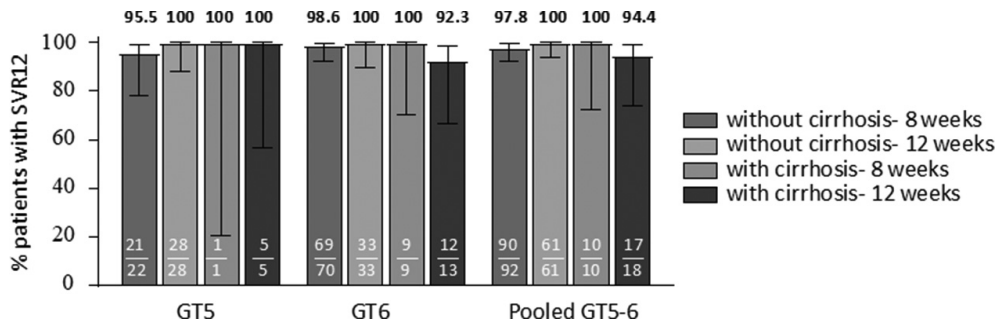

Abstract IDDF2019-ABS-0211 Figure 1 Efficacy with G/P in GT5/GT6 patients 